\title{
Intestinal Metaplasia of Gastric Mucosa
}

National Cancer Institute

\section{Source}

National Cancer Institute. Intestinal Metaplasia of Gastric Mucosa. NCI Thesaurus. Code C3956.

A morphologic finding indicating the replacement of part of the gastric mucosal epithelium with intestinal-type epithelium. Using immunohistochemical staining, gastric intestinal metaplasia is classified as complete or incomplete. Complete intestinal metaplasia is defined by the decreased expression of gastric mucins and expression of the intestinal mucin MUC2. Incomplete intestinal metaplasia is defined by the coexpression of both gastric mucins and MUC2. 